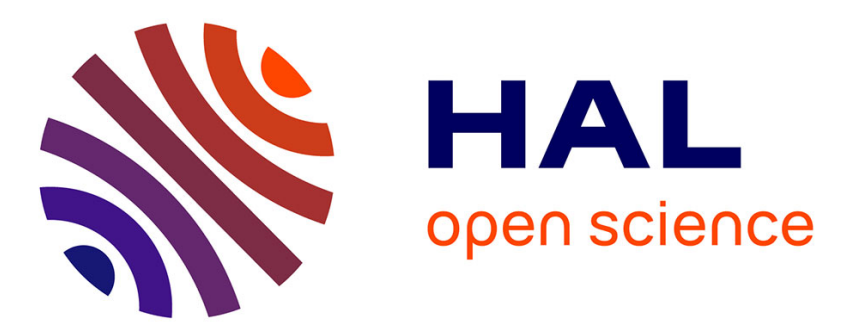

\title{
A Support System for ECG Segmentation Based on Hidden Markov Models
}

Julien Thomas, Cédric Rose, François Charpillet

\section{To cite this version:}

Julien Thomas, Cédric Rose, François Charpillet. A Support System for ECG Segmentation Based on Hidden Markov Models. 29th Annual International Conference of the IEEE Engineering in Medicine and Biology Society - IEEE EMBC 2007, Aug 2007, Lyon, France. 4 p. inria-00170988

\section{HAL Id: inria-00170988 \\ https://hal.inria.fr/inria-00170988}

Submitted on 11 Sep 2007

HAL is a multi-disciplinary open access archive for the deposit and dissemination of scientific research documents, whether they are published or not. The documents may come from teaching and research institutions in France or abroad, or from public or private research centers.
L'archive ouverte pluridisciplinaire HAL, est destinée au dépôt et à la diffusion de documents scientifiques de niveau recherche, publiés ou non, émanant des établissements d'enseignement et de recherche français ou étrangers, des laboratoires publics ou privés. 


\title{
A Support System for ECG Segmentation Based on Hidden Markov Models
}

\author{
Julien Thomas, Cédric Rose, François Charpillet
}

\begin{abstract}
Pharmaceutic studies require to analyze thousands of ECGs in order to evaluate the side effects of a new drug. In this paper we present a new support system based on the use of probabilistic models for automatic ECG segmentation. We used a bayesian HMM clustering algorithm to partition the training base, and we improved the method by using a multichannel segmentation. We present a statistical analysis of the results where we compare different automatic methods to the segmentation of the cardiologist as a gold standard.
\end{abstract}

\section{INTRODUCTION}

During the development of a new drug, the Food and Drug Administration (FDA) and some other health organizations require to make studies in order to highlight undesirable side effects on various physiological indicators [1]. One of these side effects is the impact of the drug on the cardiovascular system. The best way to prevent cardiac injuries is to record thousands of ECGs and analyze them.

The first step of ECG analysis, the ECG reading, is the measurement of several intervals which represent specific phases of the cardiac cycle. Then their evolution is studied in order to evaluate the impact on the heart according to various drug amounts. Currently this reading is carried out while locating markers for the beginning and the end of each ECG wave in a manual way by operators, trained with ECG reading, then it is validated by cardiologists. This is the segmentation step.

Several commercial softwares, based on ad hoc algorithms, have been developed in order to help cardiologists to segment large amounts of ECGs. But these softwares do not provide a precise segmentation, and repetitive corrections have to be made. Using probabilistic models, such as hidden Markov models (HMM), in a decision support tool makes it possible not only to imitate the behavior of a cardiologist, but also to learn a reasoning mode and to provide its own analysis of the problem.

This paper is organized as follows. Section 2 presents previous works on automated ECG segmentation. Section 3 introduces our proposal. Sections 4 and 5 describe how to use multiple HMMs combined with the Haar wavelet representation for ECG segmentation. Section 6 describes the experimental method and we discuss about the results.

\section{PREVIOUS WORK ON ECG SEGMENTATION}

An extensive review and comparison of QRS detection algorithms can be found in [2]. Many approaches consist in

J. Thomas is with Cardiabase, 78 avenue du XXème Corps, 54000 Nancy, France jthomaseloria.fr

C. Rose and F. Charpillet are with INRIA-LORIA, B.P.239, 54506 Vandoeuvre-lès-Nancy, France $\{$ rose, charpillet\}@loria.fr comparing signal features to a threshold. In spite of the good results on QRS detection, interval analysis remains a difficult task for these threshold based methods. The use of wavelets for ECG delineation has been studied in [3] and [4]. These works show that the analysis of wavelet coefficients can give better results than the direct analysis of the ECG voltage curve. Frequency domain filtration is implicitly performed when working with the wavelet transform. The direction changes in the signal can be detected using the Haar wavelet transform allowing to find the position of the different ECG intervals. Hidden Markov tree models have been applied in [5] to the segmentation of ECG signals encoded with the discrete wavelet transform. In [6] semi-Markov models with explicit state duration modelling were proposed for ECG interval analysis. The accuracy of ECG features detection using these models compares favorably with the previous methods. One of the advantages of probabilistic models over traditional methods is that a confidence measure for each segmented signal is given by the log likelihood of the observed signal given the model.

\section{OUR PROPOSAL}

Throughout the studies, thousands of ECGs are recorded and require to be analyzed. Ad-hoc algorithms, used in commercial softwares, are not very flexible and are not able to adapt themselves if problematic ECGs need to be segmented.

The purpose of our tool is not to replace the cardiologist intervention for the ECGs analysis. We want to give him more useful information than ad-hoc algorithms currently used. Our proposal for performing an accurate segmentation is to train the support system based on the expertise of cardiologists. Cardiologists can correct proposed segmentations and the system can refine its training. We propose a real interaction between the cardiologists and our support system.

Another essential objective of this support system is to ensure a reproducibility of the segmentation since the ECG analysis consists partly in the measurment of the evolution of the intervals among drug amounts.

\section{TOOLS}

Our method is based on the Haar wavelet transform for representation of ECGs, on the one hand, and continuous hidden Markov models for data analysis, on the other hand.

\section{A. Wavelet representation}

Models, such as HMMs, require the most relevant information in order to be efficient. In order to highlight non 
stationary properties of an ECG, we used the Continuous Wavelet Transform (CWT) [7]:

$$
\gamma(\tau, s)=\int_{-\infty}^{+\infty} f(t) \frac{1}{\sqrt{|s|}} \overline{\psi\left(\frac{t-\tau}{s}\right)} d t
$$

The CWT is performed using the convolution between the signal $f$ and the time-localized mother wavelet $\psi$, which will be translated by a factor $\tau$ and dilated by a factor $s$. The scale factor plays an important role in this wavelet transform and can be seen as the resolution of a map.

The mother wavelet selected here is the Haar wavelet, for its particular shape which allows to locate singularities precisely:

$$
\psi(t)= \begin{cases}1 & \text { if } 0 \leq t<\frac{1}{2} \\ -1 & \text { if } \frac{1}{2} \leq t<1 \\ 0 & \text { otherwise }\end{cases}
$$

\section{B. Hidden Markov Models}

Stochastic models have been successfully applied for signal modeling and work very well for building recognition systems. More precisely, HMMs are often used for speech segmentation [8] for example.

A HMM is a stochastic automaton representing a markovian process. A continuous density HMM of size $N$ can be fully described by the triplet $\lambda=(A, B, \Pi)$ where:

- $A$ is a $(N, N)$ matrix of transition probabilities such as $A(i, j)=P\left(S_{t}=j \mid S_{t-1}=i\right)$,

- $B$ is a set of $N$ observation probability density functions $B=\left(B_{1}, . ., B_{N}\right)$ where $B_{i}=P\left(O_{t} \mid S_{t}=i\right)$,

- $\Pi$ is an initial probability distribution over the HMM states: $\Pi=P\left(S_{0}\right)$.

The training of the model consists in discovering the parameters $(A, B, \Pi)$ from the observation of sample signals. The recognition task consists in finding the model which corresponds the most likely to a signal among a set of candidate models and in discovering the hidden state sequence corresponding to the signal.

\section{USING HMM FOR ECG SEGMENTATION}

ECG segmentation is a recognition problem. We need to recognize and precisely localize the different waves of the cardiac cycle inside the signal. Our approach for segmentation consists in building a model $\Lambda$ of the ECG, and in using the most likely state sequence for a given observation sequence in order to find the wave transitions.

This method allows us to learn the way of segmenting an ECG from an expert. The resulting model can be more or less specialized according to the variety of training cases. A specialized model will result in an accurate segmentation on cases close to the training base, but will cover a limited number of cases.

An ECG is split in ECG complexes, which are defined as a waveform succession representing one specific cardiac cycle period. The parameters of the model are learned from reference ECG complexes, segmented by a cardiologist. For our experimentation we used a data collection of 1800 properly segmented ECG complexes.

\section{A. Wave models}

As represented in figure 1, the ECG complex is split into 6 sub-waves (Base1, P, Base2, QRS, T, Base3) and a specific wave model is trained for each segment.

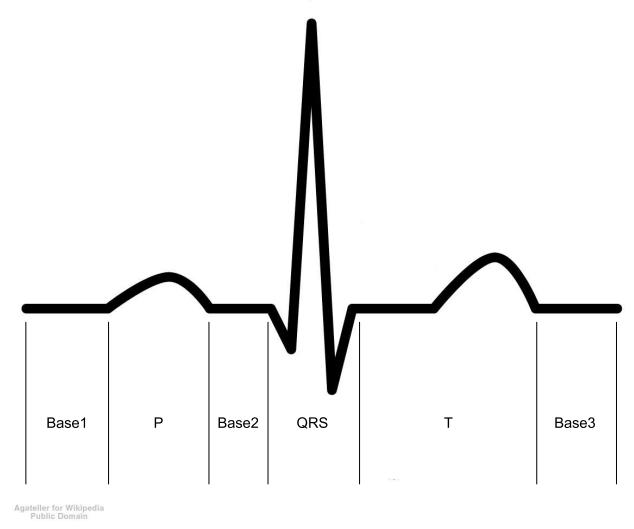

Fig. 1. ECG sub waves.

Each wave of the ECG complex is represented by a 5 states left-right continuous density HMM. Six different HMMs are trained : $\lambda_{\text {Base } 1}, \lambda_{P}, \lambda_{\text {Base } 2}, \lambda_{Q R S}, \lambda_{T}, \lambda_{\text {Base } 3}$.

Multi-dimension gaussian distributions are used as probability density functions for the observations computed by the wavelet transform.

\section{B. Models Aggregation}

During the training process, the models representing each wave are trained independently. However these six models are ineffective if they are used individually. In order to segment an ECG complex, it is necessary to build a model which represents this complex in his globality. This whole model $\Lambda$ results simply from the concatenation of the HMMs of each wave.

The resulting global HMM can be seen like a hierarchical HMM implementing a model $\Lambda$, which represents the whole ECG complex, broken up into sub models, which represent each wave. The model is composed of six states, named Base1, P, Base2, QRS, T, Base3. These six states do not represent the waves which are associated for them but sub models $\lambda_{\text {Base } 1}, \lambda_{P}, \lambda_{\text {Base } 2}, \lambda_{Q R S}, \lambda_{T}, \lambda_{\text {Base } 3}$ representing each wave.

The outgoing probability of each model is used as probability of transition to the next wave.

\section{Multi-HMM approach}

To be able to segment unspecified ECGs with high accuracy, we implemented a multi-HMM approach. This method consists in performing a bayesian clustering of the training base [9]. The training base is divided in $K$ classes of ECGs which have similarities and $K$ HMMs are trained and exploited to provide great generalization capabilities and high accuracy at the same time.

This HMM clustering algorithm is simply a variation of the K-Means algorithm, where the clusters are defined by HMMs rather than by centers in the data space. For a given 
class, the notion of distance to the center is replaced by the likelihood $P\left(O_{i} \mid \Lambda_{j}\right)$ of the observation sequence. The number of classes $K$ is fixed arbitrarily.

The goal is to maximize the global likelihood of the training base given the HMMs. The likelihood of a particular observation sequence is given by:

$$
P\left(O_{i} \mid \Lambda_{1}, . ., \Lambda_{K}\right)=\max _{j \in[1 . . K]} P\left(O_{i} \mid \Lambda_{j}\right)
$$

The HMM structure used in the clustering process is the same as the one used for ECG segmentation. As a results the cluster HMMs $\left(\Lambda_{1}, . ., \Lambda_{K}\right)$ can directly be used for ECG segmentation. In order to analyze a new signal $O$, we simply need to select the model with the highest likelihood (the model $\Lambda_{i}$ that maximizes $P\left(O \mid \Lambda_{i}\right)$ ).

\section{Segmentation of multi-channel ECGs}

An ECG is recorded on 12 leads, which show the same electric activity under 12 different angles [10]. One particular HMM is trained for each lead, providing 12 choices to position a marker. We found that the median value is the best method to obtain accurate segmentation of an ECG. Aberrant values, due to bad recordings for example, can be filtered out by using this method.

\section{EXPERIMENTAL METHOD AND RESULTS}

\section{A. Methodology}

The training base is constituted of 1800 ECG complexes, each corresponding to a different patient, manually segmented by two experimented senior cardiologists. All this complexes come from the Cardiabase company database.

Two approaches are considered: a method for which the training relates to the whole of these recordings, called the "generic" approach, and a clustering method, based on KMeans, with $K=10$ classes referred to as the "cluster" approach.

To evaluate their efficiency, these 2 segmentations are compared to the automatic segmentation of the ad hoc algorithm, and to the manual segmentation of the cardiologist considered as the gold standard. The statistical analysis is carried out by a paired-sample Student's t-test $(\alpha=$ 0.05) [11]. These 4 segmentation methods are applied on 173 ECG complexes.

Statistical analysis are performed with SAS software (version 9.1; SAS Institute Inc., Cary, NC).

\section{B. Comparative results and discussion}

For the 3 most important intervals (PR interval, QRS complex and QT interval), 2 tables show on the one hand a descriptive statistical analysis of the 4 segmentation methods, and on the other hand a comparison of the different methods with the paired-samples averages. All the durations are expressed in milliseconds.

Compared with the ad hoc algorithm, the "cluster" approach segmentation is closer to the cardiologist results. Especially for PR and QT intervals, the mean and median

\begin{tabular}{|l|c|c|c|c|c|}
\hline PR & Mean & Min. & Max. & Median & $\mathrm{SD}^{1}$ \\
\hline Ad hoc alg. & 160.6 & 110 & 408 & 158 & 33.3 \\
\hline Cardiologist & 159.2 & 114 & 204 & 158 & 19.0 \\
\hline Generic & 170.1 & 119 & 370 & 166 & 31.7 \\
\hline Cluster & 161.9 & 120 & 210 & 161 & 18.6 \\
\hline
\end{tabular}

TABLE I

PR INTERVAL STATISTICAL ANALYSIS.

\begin{tabular}{|l|c|c|c|c|c|}
\hline$\Delta$ PR & Mean & SD & Min. & Max. & p value \\
\hline Cardio-Ad hoc & -1.3 & 28.0 & -254 & 36 & 0.5296 \\
\hline Cardio-Generic & -10.9 & 27.3 & -233 & 18 & 0.0001 \\
\hline Cardio-Cluster & -2.7 & 5.8 & -26 & 21 & 0.0001 \\
\hline Cluster-Ad hoc & 1.3 & 28.3 & -258 & 32 & 0.5322 \\
\hline Generic-Ad hoc & 9.5 & 39.3 & -255 & 233 & 0.0016 \\
\hline Cluster-Generic & 8.2 & 25.8 & -7 & 219 & 0.0001 \\
\hline
\end{tabular}

TABLE II

COMPARISON OF DIFFERENT METHODS FOR PR INTERVAL.

\begin{tabular}{|l|c|c|c|c|c|}
\hline QRS & Mean & Min. & Max. & Median & SD $^{1}$ \\
\hline Ad hoc alg. & 84.2 & 64 & 122 & 84 & 9.5 \\
\hline Cardiologist & 95.5 & 76 & 116 & 96 & 8.3 \\
\hline Generic & 89.3 & 76 & 107 & 89 & 6.1 \\
\hline Cluster & 88.3 & 69 & 109 & 87 & 6.9 \\
\hline
\end{tabular}

TABLE III

QRS COMPLEX STATISTICAL ANALYSIS.

\begin{tabular}{|l|c|c|c|c|c|}
\hline$\Delta$ QRS & Mean & SD & Min. & Max. & p value \\
\hline Cardio-Ad hoc & 11.3 & 7.2 & -16 & 32 & 0.0001 \\
\hline Cardio-Generic & 6.1 & 6.4 & -15 & 26 & 0.0001 \\
\hline Cardio-Cluster & 7.1 & 6.9 & -12 & 30 & 0.0001 \\
\hline Cluster-Ad hoc & 4.1 & 8.0 & -25 & 24 & 0.0001 \\
\hline Generic-Ad hoc & 5.2 & 7.2 & -21 & 25 & 0.0001 \\
\hline Cluster-Generic & 1.0 & 4.2 & -20 & 10 & 0.0018 \\
\hline
\end{tabular}

TABLE IV

COMPARISON OF DIFFERENT METHODS FOR QRS COMPLEX.

\begin{tabular}{|l|c|c|c|c|c|}
\hline QT & Mean & Min. & Max. & Median & SD $^{1}$ \\
\hline Ad hoc alg. & 396.5 & 306 & 454 & 398 & 25.8 \\
\hline Cardiologist & 400.5 & 330 & 474 & 402 & 25.6 \\
\hline Generic & 403.1 & 339 & 483 & 404 & 26.3 \\
\hline Cluster & 399.0 & 330 & 481 & 400 & 25.3 \\
\hline
\end{tabular}

TABLE V

QT INTERVAL STATISTICAL ANALYSIS.

\begin{tabular}{|l|c|c|c|c|c|}
\hline$\Delta$ QT & Mean & SD & Min. & Max. & p value \\
\hline Cardio-Ad hoc & 4.0 & 7.0 & -18 & 24 & 0.0001 \\
\hline Cardio-Generic & -2.5 & 16.3 & -142 & 36 & 0.0442 \\
\hline Cardio-Cluster & 1.5 & 8.8 & -30 & 27 & 0.0217 \\
\hline Cluster-Ad hoc & 2.5 & 9.6 & -29 & 34 & 0.0009 \\
\hline Generic-Ad hoc & 6.5 & 17.9 & -54 & 166 & 0.0001 \\
\hline Cluster-Generic & 4.1 & 14.2 & -25 & 142 & 0.0002 \\
\hline
\end{tabular}

TABLE VI

COMPARISON OF DIFFERENT METHODS FOR QT INTERVAL.

\footnotetext{
${ }^{1}$ Standard deviation
} 
are very close to the cardiologist values. Despite higher computation time than for the "generic" approach, the multiHMM one gives a more accurate segmentation. With the "generic" approach the dispersion for this two intervals is higher.

For the QRS complex however, both the "generic" and "cluster" approaches are not efficient enough: the QRS complex durations obtained by these two methods are lower than those obtained by the cardiologist. In fact the QRS begin marker is at the same time the end of the PR segment and the beginning of the QRS complex. The PR segment of a ECG lead is sometimes very short. The states number for the PR segment model $\left(\lambda_{B a s e 2}\right)$ could be decreased so the QRSonset marker could be detect earlier. We expect that the QRS complex durations will be closer to the cardiologist ones.

Even though our algorithm has some problems segmenting the QRS complex, we have much better results with the "cluster" approach than the ad hoc algorithm for the three intervals.

\section{FUTURE WORKS}

To optimize the segmentation of the 3 intervals and more particularly for the QRS complex, several improvements are envisaged. Initially, the easiest way to improve the segmentation is to enrich the training of the HMMs by increasing the number of ECG complexes provided to the training base. Then, we plan to determine the optimal states number for each wave model by using a criterion such as bayesian information criterion (BIC). The next step for clustering experiment will be to use BIC to define the best number of clusters, so this number will no longer be fixed arbitrarily. The adaptation for a specific subject could also be considered in the case of a large pool of ECGs per patient.

This segmentation tool could be included in a complete support system providing analysis for ventricular arrhythmia, atrial fibrillation or QT duration prolongation (to prevent the torsade de pointes phenomena.

\section{CONCLUSION}

We have presented a machine learning support system to ECG segmentation. We trained continuous density HMMs on ECGs segmented by cardiologists. The Haar continuous wavelet transform was used to encode the ECGs. A "cluster" approach based on the K-Means clustering algorithm was implemented in order to generate ECGs classes among the training base. Because of the specialization of each class, we have obtained segmentations nearer to one of the cardiologist than with the "generic" approach.

A set of 1800 ECG complexes segmented by a cardiologist was used for the training of the models. The accuracy of the "generic" and the "cluster" methods was measured on 173 ECG complexes using the cardiologist segmentation as a reference. The results were also compared to a commercial ad hoc algorithm. The "cluster" approach gives significantly better results than the "generic" method but presents the drawback of having a higher computation cost.

\section{ACKNOWLEDGMENTS}

This work is the result of a collaboration between the LORIA (Laboratoire lorrain de Recherche en Informatique et ses Applications) and the Cardiabase company. We thank the cardiologist Pascal Voiriot, the statistician Sahar Bayat and the IT manager Matthieu Saunier from Cardiabase for their contribution to this work.

\section{REFERENCES}

[1] Food And Drug Administration. General Considerations For The Clinical Evaluation Of Drugs. U.S. Department Of Health, Education And Welfare, 1997.

[2] B.-U. Köhler, C. Hennig, and R. Orglmeister. The principles of software QRS detection. Engineering in Medicine and Biology Magazine, IEEE, 2002.

[3] J. P. Martinez, R. Almeida, S. Olmos, A. P. Rocha, and P. Laguna. A wavelet-based ECG delineator: Evaluation on standard databases. In IEEE TRANSACTIONS ON BIOMEDICAL ENGINEERING BME, 2004.

[4] A. Khawaja, S. Sanyal, and O Dössel. A wavelet-based multichannel ECG delineator. In The 3rd European Medical and Biological Engineering Conference, 2005.

[5] S. Graja and J.-M. Boucher. Markov models for automated ECG interval analysis. In WISP 2003: IEEE International Symposium on Inteligent Signal Processing, pages 105-109, 2003.

[6] N. P. Hughes, L. Tarassenko, and S. J. Roberts. Markov models for automated ECG interval analysis. In S. Thrun, L. Saul, and B. Schoelkopf, editors, Advances in Neural Information Processing Systems 16, Cambridge, MA, 2004. MIT Press.

[7] I. Daubechies. Ten lectures on Wavelet. SIAM, Philadelphia, 1992.

[8] L. R. Rabiner. A tutorial on hidden markov models and selected applications in speech recognition. In Proceedings of the IEEE, pages 257-286, 1989.

[9] C. Li and G. Biswas. A bayesian approach to temporal data clustering using hidden markov models. In Proceedings of the Seventeenth International Conference on Machine Learning, pages 543-550, 2000.

[10] B. Aehlert. ECG's Made Easy 2nd Edition. C.V. Mosby, St. Louis, 2001.

[11] M. Abramowitz and I. A. Stegun. Handbook of Mathematical Functions with Formulas, Graphs, and Mathematical Tables. Dover, New York, 1964. 